\title{
Table of criterion values $(\beta)$ used in signal detection theory
}

\author{
RICK M. GARDNER, SUZANNE DALSING, BETH REYES, and SHIRLEY BRAKE \\ University of Southern Colorado, Pueblo, Colorado
}

\begin{abstract}
A table of criterion values for use with the theory of signal detection (TSD) is presented. Criterion values $(\beta)$ are presented for varying proportions of hits and false alarms. A brief explanation of signal detection theory is given, and the measures for sensory sensitivity $\left(d^{\prime}\right)$ and response criterion $(\beta)$ are described.
\end{abstract}

The theory of signal detection (TSD) originally proposed by Tanner and Swets (1954), has served as an alternative to the classical psychophysical methodologies in a variety of psychophysical studies and has more recently been used in analyzing data in a variety of areas as diverse as memory and medical diagnosis.

Signal detection theory uses psychophysical methodology, which allows separation of sensory and nonsensory factors. Briefly, this theory assumes the process of signal detection to be a choice between two Gaussian variables. One variable, having a mean equal to zero, is associated with noise alone; the other variable has a mean equal to signal plus noise. In the most common detection problem, the observer decides, on the basis of an observation that is a sample of one of the populations, which of the two alternatives existed during the observation interval. The particular decision made depends upon whether or not the observation exceeds a criterion value $(\beta)$. The criterion, in turn, depends upon the observer's detection goal and upon information available about relevant parameters of the detection situation. The sensitivity of the decision-making process is a function of the variable $\mathrm{d}^{\prime}$, which is equal to the distance between the noise and signal-plus-noise distribution and which is monotonically related to the signal strength.

In any signal detection analysis, two values are of interest: some measure of sensory sensitivity and an independent measure of response criterion. Values of d' can be determined directly for any combination of false alarms and hit proportions by using a table developed by Elliot (1964).

Despite the widespread use of TSD, no concomitant table for determining criterion values $(\beta)$ from false-alarm and hit proportions has been published. Beta is the ratio of the ordinate of the $\mathrm{SN}$ distribution at the criterion to the ordinate of the $\mathrm{N}$ distribution at the criterion, as follows:

$$
\beta=\left(\mathrm{f}_{\mathrm{sn}(\mathrm{x})} \text { at criterion }\right) /\left(\mathrm{f}_{\mathrm{n}(\mathrm{x})} \text { at criterion }\right) .
$$

The authors' mailing address is: Department of Psychology, University of Southern Colorado, Pueblo, CO 81005.
The ordinate of $f_{n(x)}$ gives the probability of an observation's occurring when only noise is presented. Similarly, the ordinate of $f_{s n(x)}$ gives the likelihood of an observation's occurring when a signal is presented. Beta, then, is a likelihood ratio that provides the observer with a basis for making a decision, since it expresses the likelihood of an observation's coming from the SN distribution relative to the likelihood of its coming from the noise distribution.

Like $\mathrm{d}^{\prime}$, the value of $\beta$ can be calculated from a pair of hit and false-alarm rates. The ordinate of the $\mathrm{N}$ distribution at criterion can be estimated as the ordinate value given in the table of the normal distribution that corresponds to the false-alarm rate. Similarly, the ordinate of the SN distribution at criterion is obtained by converting the hit rate into the ordinate value on the normal distribution curve. Gescheider (1976, p. 64) illustrated this procedure in detail. An observer who is maximizing hits while minimizing false alarms will have a $\beta$ that is equal to 1.00 . A value of $\beta$ below 1.00 represents a lax criterion, such that the observer will be liberal about reporting signals; a high value of $\beta$ (i.e., above 1.00) represents a strict criterion, such that the observer will be conservative about reporting signals.

Table 1 gives the value of $\beta$ for each corresponding pair of hit and false-alarm proportions. The values along the top of Table 1 represent the false-alarm rate $[p(y e s \mid n)]$, whereas the values on the side represent the hit rate [p(yes $\mid \mathrm{sn})$ ]. Users of Table 1 are cautioned against averaging $\beta$ values over subjects, trial blocks, etc., because the distribution is asymmetric. Average values of a criterion should usually be calculated by the exponential of the average log likelihood ratio.

Use of $\mathrm{d}^{\prime}$ and $\beta$ values typically assumes that the noise and signal-plus-noise curves are normally distributed with equal variances. In cases in which these assumptions are not met, an alternative measure of sensitivity, $A^{\prime}$, was proposed by Pollack (1976). $A^{\prime}$ is a nonparametric analogue to $\mathrm{d}$ ' and is based on the area under a kind of "average" receiver-operating-characteristic (ROC) curve. Snodgrass (1984) computed a nonparametric response criterion index corresponding to this measure, which she 
Table 1

Values of Response Criterion ( $\beta$ for Varying Proportions of False Alarms [ $p(y e s \mid n)]$ and Hits [p(yes $\mid \operatorname{sn})]$

Hits

[p(yes $\mid s n)]$

\begin{tabular}{|c|c|c|c|c|c|c|c|c|c|c|}
\hline & .01 & .02 & .03 & .04 & .05 & .06 & .07 & .08 & .09 & . 10 \\
\hline .01 & 1.00 & 0.55 & 0.39 & 0.31 & 0.26 & 0.22 & 0.20 & 0.18 & 0.16 & 0.15 \\
\hline .02 & 1.82 & 1.00 & 0.71 & 0.56 & 0.47 & 0.41 & 0.36 & 0.33 & 0.30 & 0.28 \\
\hline .03 & 2.55 & 1.40 & 1.00 & 0.79 & 0.66 & 0.57 & 0.51 & 0.46 & 0.42 & 0.39 \\
\hline .04 & 3.24 & 1.78 & 1.27 & 1.00 & 0.83 & 0.72 & 0.64 & 0.58 & 0.53 & 0.49 \\
\hline .05 & 3.88 & 2.13 & 1.52 & 1.20 & 1.00 & 0.87 & 0.77 & 0.69 & 0.64 & 0.59 \\
\hline .06 & 4.48 & 2.46 & 1.75 & 1.38 & 1.15 & 1.00 & 0.89 & 0.80 & 0.73 & 0.68 \\
\hline .07 & 5.05 & 2.77 & 1.98 & 1.56 & 1.30 & 1.13 & 1.00 & 0.90 & 0.83 & 0.77 \\
\hline .08 & 5.59 & 3.107 & 2.19 & 1.73 & 1.44 & 1.25 & 1.11 & 1.00 & 0.91 & 0.85 \\
\hline .09 & 6.11 & 3.36 & 2.39 & 1.89 & 1.57 & 1.36 & 1.21 & 1.69 & 1.00 & 0.93 \\
\hline .10 & 6.59 & 3.62 & 2.58 & 2.04 & 1.70 & 1.47 & 1.31 & 1.18 & 1.08 & 1.06 \\
\hline .11 & 7.07 & 3.88 & 2.77 & 2.18 & 1.82 & 1.58 & 1.40 & 1.27 & 1.16 & 1.07 \\
\hline .12 & 7.52 & 4.13 & 2.95 & 2.32 & 1.94 & 1.68 & 1.49 & 1.35 & 1.23 & 1.14 \\
\hline .13 & 7.95 & 4.37 & 3.12 & 2.46 & 2.05 & 1.78 & 1.58 & 1.42 & 1.30 & 1.21 \\
\hline .14 & 8.37 & 4.60 & 3.28 & 2.59 & 2.16 & 1.87 & 1.66 & 1.50 & 1.37 & 1.27 \\
\hline . 15 & 8.76 & 4.82 & 3.43 & 2.71 & 2.26 & 1.96 & 1.74 & 1.57 & 1.43 & 1.33 \\
\hline .16 & 9.15 & 5.03 & 3.58 & 2.83 & 2.36 & 2.04 & 1.81 & 1.64 & 1.50 & 1.39 \\
\hline .17 & 9.51 & 5.23 & 3.73 & 2.94 & 2.45 & 2.12 & 1.88 & 1.70 & 1.56 & 1.44 \\
\hline .18 & 9.86 & 5.42 & 3.86 & 3.05 & 2.54 & 2.20 & 1.95 & 1.77 & 1.61 & 1.50 \\
\hline .19 & 10.20 & 5.61 & 4.06 & 3.15 & 2.63 & 2.28 & 2.02 & 1.83 & 1.67 & 1.55 \\
\hline .211 & 10.52 & 5.78 & 4.12 & 3.25 & 2.71 & 2.35 & 2.08 & 1.88 & 1.72 & 1.60 \\
\hline .21 & 10.83 & 5.95 & 4.24 & 3.35 & 2.79 & 2.42 & 2.15 & 1.94 & 1.77 & 1.64 \\
\hline .22 & 11.13 & 6.12 & 4.36 & 3.44 & 2.87 & 2.49 & 2.20 & 1.99 & 1.82 & 1.69 \\
\hline .23 & 11.41 & 6.27 & 4.47 & 3.53 & 2.94 & 2.55 & 2.26 & 2.04 & 1.87 & 1.73 \\
\hline .24 & 11.69 & 6.42 & 4.58 & 3.61 & 3.01 & 2.61 & 2.31 & 2.09 & 1.91 & 1.77 \\
\hline .25 & 11.95 & 6.57 & 4.68 & 3.69 & 3.08 & 2.67 & 2.37 & 2.14 & 1.96 & 1.81 \\
\hline .26 & 12.20 & 6.70 & 4.78 & 3.77 & 3.14 & 2.72 & 2.42 & 2.18 & 2.06 & 1.85 \\
\hline .27 & 12.43 & 6.83 & 4.87 & 3.84 & 3.20 & 2.78 & 2.46 & 2.23 & 2.04 & 1.89 \\
\hline .28 & 12.65 & 6.95 & 4.96 & 3.91 & 3.26 & 2.83 & 2.51 & 2.27 & 2.07 & 1.92 \\
\hline .29 & 12.86 & 7.07 & 5.04 & 3.97 & 3.32 & 2.87 & 2.55 & 2.30 & 2.11 & 1.95 \\
\hline . 310 & 13.97 & 7.18 & 5.12 & 4.04 & 3.37 & 2.92 & 2.59 & 2.34 & 2.14 & 1.98 \\
\hline .31 & 13.26 & 7.29 & 5.20 & 4.10 & 3.42 & 2.96 & 2.63 & 2.37 & 2.17 & 2.01 \\
\hline .32 & 13.44 & 7.39 & 5.27 & 4.15 & 3.47 & 3.00 & 2.66 & 2.41 & 2.200 & 2.04 \\
\hline .33 & 13.61 & 7.48 & 5.33 & 4.21 & 3.51 & 3.04 & 2.70 & 2.44 & 2.23 & 2.06 \\
\hline .34 & 13.77 & 7.57 & 5.40 & 4.26 & 3.55 & 3.08 & 2.73 & 2.47 & 2.25 & 2.09 \\
\hline .35 & 13.92 & 7.65 & 5.46 & 4.30 & 3.59 & 3.11 & 2.76 & 2.49 & 2.28 & 2.11 \\
\hline .36 & 14.06 & 7.73 & 5.51 & 4.34 & 3.63 & 3.14 & 2.79 & 2.52 & 2.30 & 2.13 \\
\hline .37 & 14.200 & 7.80 & 5.56 & 4.39 & 3.66 & 3.17 & 2.81 & 2.54 & 2.32 & 2.15 \\
\hline .38 & 14.31 & 7.87 & 5.61 & 4.42 & 3.69 & 3.20 & 2.83 & 2.56 & 2.34 & 2.17 \\
\hline .39 & 14.42 & 7.93 & 5.65 & 4.46 & 3.72 & 3.22 & 2.86 & 2.58 & 2.36 & 2.19 \\
\hline .40 & 14.53 & 7.98 & 5.69 & 4.49 & 3.74 & 3.24 & 2.88 & 2.60 & 2.38 & 2.260 \\
\hline .41 & 14.61 & 8.63 & 5.72 & 4.51 & 3.77 & 3.26 & 2.89 & 2.62 & 2.39 & 2.22 \\
\hline .42 & 14.70 & 8.08 & 5.76 & 4.54 & 3.79 & 3.28 & 2.91 & 2.63 & 2.41 & 2.23 \\
\hline .43 & 14.77 & 8.12 & 5.78 & 4.56 & 3.81 & 3.30 & 2.92 & 2.64 & 2.42 & 2.24 \\
\hline .44 & 14.83 & 8.15 & 5.81 & 4.58 & 3.82 & 3.31 & 2.94 & 2.65 & 2.43 & 2.25 \\
\hline .45 & 14.88 & 8.18 & 5.83 & 4.669 & 3.84 & 3.32 & 2.95 & 2.66 & 2.44 & 2.26 \\
\hline .46 & 14.92 & 8.280 & 5.85 & 4.61 & 3.85 & 3.33 & 2.96 & 2.67 & 2.44 & 2.26 \\
\hline .47 & 14.95 & 8.22 & 5.86 & 4.62 & 3.85 & 3.34 & 2.96 & 2.68 & 2.45 & 2.27 \\
\hline .48 & 14.98 & 8.23 & 5.87 & 4.63 & 3.86 & 3.35 & 2.97 & 2.68 & 2.45 & 2.27 \\
\hline .49 & 14.99 & 8.24 & 5.87 & 4.63 & 3.86 & 3.35 & 2.97 & 2.68 & 2.45 & 2.27 \\
\hline .50 & 15.00 & 8.24 & 5.87 & 4.63 & 3.87 & 3.35 & 2.97 & 2.68 & 2.45 & 2.27 \\
\hline
\end{tabular}

False Alarms [p(yes|n)] 
Table 1 Continued

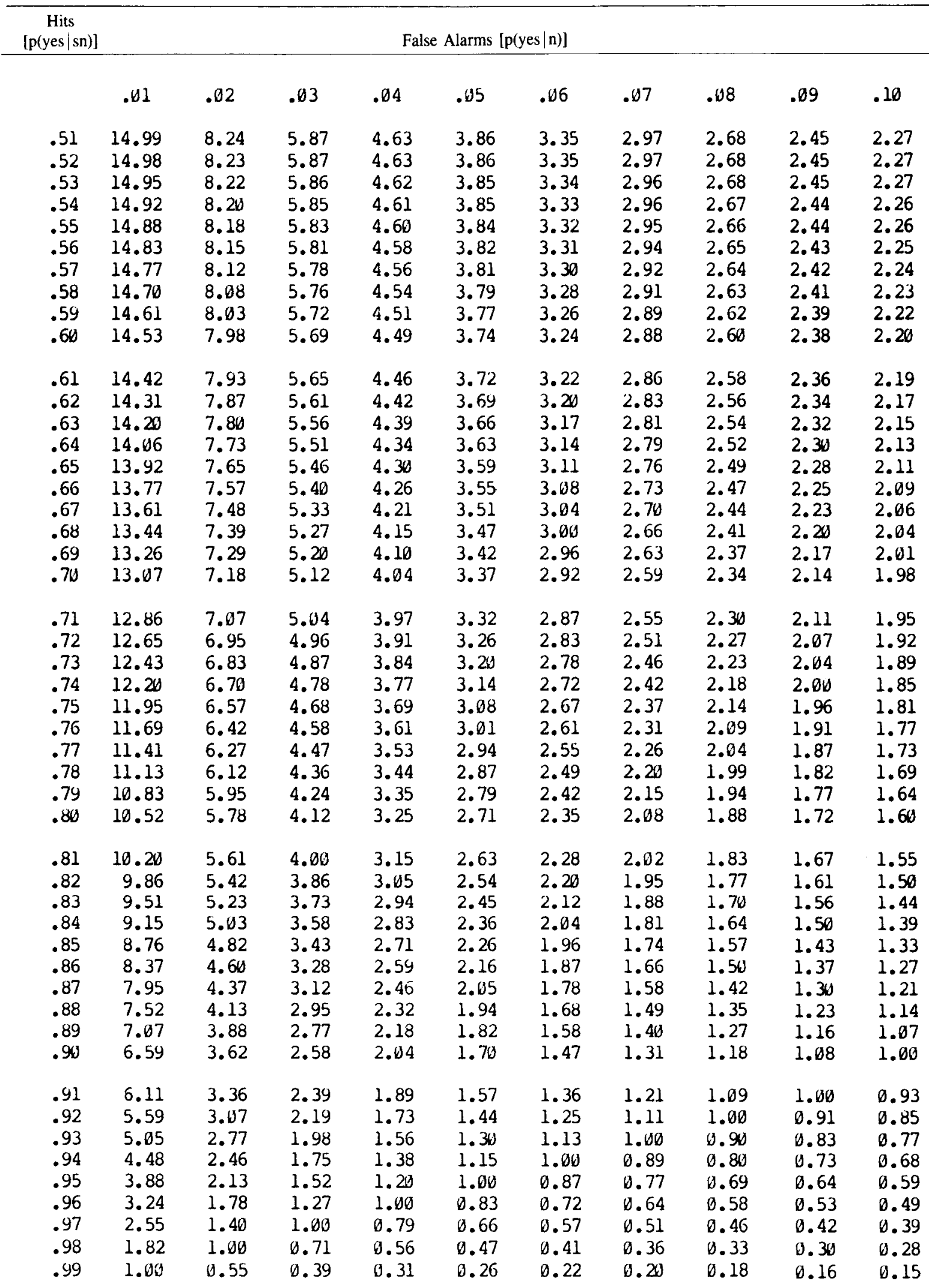


Table 1 Continued

\begin{tabular}{|c|c|c|c|c|c|c|c|c|c|c|}
\hline $\begin{array}{c}\text { Hits } \\
{[p(y e s / s n)]}\end{array}$ & & & & Fal & Alarms I & es $\mid \mathrm{n})]$ & & & & \\
\hline & .11 & .12 & .13 & .14 & .15 & .16 & .17 & .18 & .19 & .20 \\
\hline .01 & 0.14 & 0.13 & 0.13 & 0.12 & 0.11 & 0.11 & 0.11 & 0.10 & 0.10 & 0.10 \\
\hline .02 & 0.26 & 0.24 & 0.23 & 0.22 & 0.21 & 0.20 & 0.19 & 0.18 & 0.18 & 0.17 \\
\hline .03 & 0.36 & 0.34 & 0.32 & 0.31 & 0.29 & 0.28 & 0.27 & 0.26 & 0.25 & 0.24 \\
\hline .04 & 0.46 & 0.43 & 0.41 & 0.39 & 0.37 & 0.35 & D. 34 & 0.33 & 0.32 & 0.31 \\
\hline .05 & 0.55 & 0.52 & 0.49 & 0.46 & 0.44 & 0.42 & 0.41 & 0.39 & 0.38 & 0.37 \\
\hline .06 & 0.63 & 0.60 & 0.56 & 0.54 & 0.51 & 0.49 & 0.47 & 0.45 & 0.44 & 0.43 \\
\hline .07 & 0.71 & 0.67 & 0.63 & 0.60 & 0.58 & 0.55 & 0.53 & 0.51 & 0.49 & 0.48 \\
\hline .08 & 10.79 & 0.74 & 0.70 & 0.67 & 0.64 & 0.61 & 0.59 & 0.57 & 0.55 & 0.53 \\
\hline .09 & 0.86 & 0.81 & 0.77 & 0.73 & 0.70 & 0.67 & 0.64 & 0.62 & 0.60 & 0.58 \\
\hline .10 & 0.93 & 10.88 & 0.83 & 0.79 & 0.75 & 0.72 & b. 69 & 0.67 & 0.65 & 0.63 \\
\hline .11 & 1.00 & 0.94 & 0.89 & 0.84 & 0.81 & 0.77 & 0.74 & 0.72 & 0.69 & 0.67 \\
\hline .12 & 1.06 & 1.00 & 0.95 & 0.90 & 0.86 & 0.82 & 0.79 & 0.76 & 0.74 & 0.71 \\
\hline .13 & 1.13 & 1.06 & 1.00 & 0.95 & 0.91 & 0.87 & 0.84 & 0.81 & 0.78 & 0.76 \\
\hline .14 & 1.18 & 1.11 & 1.05 & 1.00 & 0.95 & 0.91 & 0.88 & 0.85 & 0.82 & 0.80 \\
\hline .15 & 1.24 & 1.16 & 1.10 & 1.05 & 1.00 & 0.96 & 0.92 & 0.89 & 0.86 & 0.83 \\
\hline .16 & 1.29 & 1.22 & 1.15 & 1.09 & 1.04 & 1.00 & 0.96 & 0.93 & 0.90 & 0.87 \\
\hline .17 & 1.35 & 1.26 & 1.20 & 1.14 & 1.09 & 1.04 & 1.00 & 0.96 & 0.93 & 0.90 \\
\hline .18 & 1.40 & 1.31 & 1.24 & 1.18 & 1.13 & 1.08 & 1.04 & 1.000 & 0.97 & 0.94 \\
\hline .19 & 1.44 & 1.36 & 1.28 & 1.22 & 1.16 & 1.12 & 1.07 & 1.03 & 1.06 & 0.97 \\
\hline .200 & 1.49 & 1.40 & 1.32 & 1.26 & 1.20 & 1.15 & 1.11 & 1.07 & 1.03 & 1.00 \\
\hline .21 & 1.53 & 1.44 & 1.36 & 1.29 & 1.24 & 1.18 & 1.14 & 1.10 & 1.06 & 1.03 \\
\hline .22 & 1.58 & 1.48 & 1.40 & 1.33 & 1.27 & 1.22 & 1.17 & 1.13 & 1.09 & 1.06 \\
\hline .23 & 1.61 & 1.52 & 1.43 & 1.36 & 1.360 & 1.25 & 1.20 & 1.16 & 1.12 & 1.08 \\
\hline .24 & 1.65 & 1.55 & 1.47 & 1.40 & 1.33 & 1.28 & 1.23 & 1.18 & 1.15 & 1.11 \\
\hline .25 & 1.69 & 1.59 & 1.50 & 1.43 & 1.36 & 1.31 & 1.26 & 1.21 & 1.17 & 1.14 \\
\hline .26 & 1.73 & 1.62 & 1.53 & 1.46 & 1.39 & 1.33 & 1.28 & 1.24 & 1.200 & 1.16 \\
\hline .27 & 1.76 & 1.65 & 1.56 & 1.49 & 1.42 & 1.36 & 1.31 & 1.26 & 1.22 & 1.18 \\
\hline .28 & 1.79 & 1.68 & 1.59 & 1.51 & 1.44 & 1.38 & 1.33 & 1.28 & 1.24 & 1.20 \\
\hline .29 & 1.82 & 1.71 & 1.62 & 1.54 & 1.47 & 1.41 & 1.35 & 1.36 & 1.26 & 1.22 \\
\hline .30 & 1.85 & 1.74 & 1.64 & 1.56 & 1.49 & 1.43 & 1.37 & 1.33 & 1.28 & 1.24 \\
\hline .31 & 1.88 & 1.76 & 1.67 & 1.58 & 1.51 & 1.45 & 1.39 & 1.34 & 1.30 & 1.26 \\
\hline .32 & 1.90 & 1.79 & 1.69 & 1.61 & 1.53 & 1.47 & 1.41 & 1.36 & 1.32 & 1.28 \\
\hline .33 & 1.93 & 1.81 & 1.71 & 1.63 & 1.55 & 1.49 & 1.43 & 1.38 & 1.33 & 1.29 \\
\hline .34 & 1.95 & 1.83 & 1.73 & 1.65 & 1.57 & 1.51 & 1.45 & 1.40 & 1.35 & 1.31 \\
\hline .35 & 1.97 & 1.85 & 1.75 & 1.66 & 1.59 & 1.52 & 1.46 & 1.41 & 1.36 & 1.32 \\
\hline .36 & 1.99 & 1.87 & 1.77 & 1.68 & 1.60 & 1.54 & 1.48 & 1.43 & 1.38 & 1.34 \\
\hline .37 & 2.01 & 1.89 & 1.78 & 1.76 & 1.62 & 1.55 & 1.49 & 1.44 & 1.39 & 1.35 \\
\hline .38 & 2.03 & 1.90 & 1.80 & 1.71 & 1.63 & 1.56 & 1.50 & 1.45 & 1.40 & 1.36 \\
\hline .39 & 2.04 & 1.92 & 1.81 & 1.72 & 1.65 & 1.58 & 1.52 & 1.46 & 1.41 & 1.37 \\
\hline .40 & 2.06 & 1.93 & 1.83 & 1.74 & 1.66 & 1.59 & 1.53 & 1.47 & 1.42 & 1.38 \\
\hline .41 & 2.07 & 1.94 & 1.84 & 1.75 & 1.67 & 1.60 & 1.54 & 1.48 & 1.43 & 1.39 \\
\hline .42 & 2.08 & 1.95 & 1.85 & 1.76 & 1.68 & 1.61 & 1.55 & 1.49 & 1.44 & 1.40 \\
\hline .43 & 2.09 & 1.96 & 1.86 & 1.76 & 1.69 & 1.61 & 1.55 & 1.56 & 1.45 & 1.40 \\
\hline .44 & 2.10 & 1.97 & 1.86 & 1.77 & 1.69 & 1.62 & 1.56 & 1.50 & 1.45 & 1.41 \\
\hline .45 & 2.11 & 1.98 & 1.87 & 1.78 & 1.70 & 1.63 & 1.56 & 1.51 & 1.46 & 1.41 \\
\hline .46 & 2.11 & 1.98 & 1.88 & 1.78 & 1.70 & 1.63 & 1.57 & 1.51 & 1.46 & 1.42 \\
\hline .47 & 2.12 & 1.99 & 1.88 & 1.79 & 1.71 & 1.64 & 1.57 & 1.52 & 1.47 & 1.42 \\
\hline .48 & 2.12 & 1.99 & 1.88 & 1.79 & 1.71 & 1.64 & 1.57 & 1.52 & 1.47 & 1.42 \\
\hline .49 & 2.12 & 1.99 & 1.88 & 1.79 & 1.71 & 1.64 & 1.58 & 1.52 & 1.47 & 1.42 \\
\hline .50 & 2.12 & 1.99 & 1.89 & 1.79 & 1.71 & 1.64 & 1.58 & 1.52 & 1.47 & 1.43 \\
\hline
\end{tabular}


Table 1 Continued

\begin{tabular}{|c|c|c|c|c|c|c|c|c|c|c|}
\hline $\begin{array}{c}\text { Hits } \\
{[\mathrm{p}(\text { yes } \mid \mathrm{sn})]}\end{array}$ & & & & & Alarms & es|n)] & & & & \\
\hline & .11 & .12 & .13 & .14 & .15 & .16 & .17 & .18 & .19 & .20 \\
\hline & $\begin{array}{r}\text { or } \\
.89\end{array}$ & $\begin{array}{l}\text { or } \\
.88\end{array}$ & $\begin{array}{r}\text { or } \\
.87\end{array}$ & $\begin{array}{r}\text { or } \\
.86\end{array}$ & $\begin{array}{l}0 r \\
.85\end{array}$ & $\begin{array}{l}\text { or } \\
.84\end{array}$ & $\begin{array}{r}\text { or } \\
.83\end{array}$ & $\begin{array}{r}\text { or } \\
.82\end{array}$ & $\begin{array}{l}\text { or } \\
.81\end{array}$ & $\begin{array}{l}\text { or } \\
.80\end{array}$ \\
\hline $\begin{array}{l}.51 \\
.52 \\
.53 \\
.54 \\
.55 \\
.56 \\
.57 \\
.58 \\
.59 \\
.60\end{array}$ & $\begin{array}{l}2.12 \\
2.12 \\
2.12 \\
2.11 \\
2.11 \\
2.10 \\
2.09 \\
2.08 \\
2.07 \\
2.06\end{array}$ & $\begin{array}{l}1.99 \\
1.99 \\
1.99 \\
1.98 \\
1.98 \\
1.97 \\
1.96 \\
1.95 \\
1.94 \\
1.93\end{array}$ & $\begin{array}{l}1.88 \\
1.88 \\
1.88 \\
1.88 \\
1.87 \\
1.86 \\
1.86 \\
1.85 \\
1.84 \\
1.83\end{array}$ & $\begin{array}{l}1.79 \\
1.79 \\
1.79 \\
1.78 \\
1.78 \\
1.77 \\
1.76 \\
1.76 \\
1.75 \\
1.74\end{array}$ & $\begin{array}{l}1.71 \\
1.71 \\
1.71 \\
1.70 \\
1.70 \\
1.69 \\
1.69 \\
1.68 \\
1.67 \\
1.66\end{array}$ & $\begin{array}{l}1.64 \\
1.64 \\
1.64 \\
1.63 \\
1.63 \\
1.62 \\
1.61 \\
1.61 \\
1.60 \\
1.59\end{array}$ & $\begin{array}{l}1.58 \\
1.57 \\
1.57 \\
1.57 \\
1.56 \\
1.56 \\
1.55 \\
1.55 \\
1.54 \\
1.53\end{array}$ & $\begin{array}{l}1.52 \\
1.52 \\
1.52 \\
1.51 \\
1.51 \\
1.50 \\
1.50 \\
1.49 \\
1.48 \\
1.47\end{array}$ & $\begin{array}{l}1.47 \\
1.47 \\
1.47 \\
1.46 \\
1.46 \\
1.45 \\
1.45 \\
1.44 \\
1.43 \\
1.42\end{array}$ & $\begin{array}{l}1.42 \\
1.42 \\
1.42 \\
1.42 \\
1.41 \\
1.41 \\
1.40 \\
1.40 \\
1.39 \\
1.38\end{array}$ \\
\hline $\begin{array}{l}.61 \\
.62 \\
.63 \\
.64 \\
.65 \\
.66 \\
.67 \\
.68 \\
.69 \\
.70\end{array}$ & $\begin{array}{l}2.04 \\
2.03 \\
2.01 \\
1.99 \\
1.97 \\
1.95 \\
1.93 \\
1.90 \\
1.88 \\
1.85\end{array}$ & $\begin{array}{l}1.92 \\
1.93 \\
1.89 \\
1.87 \\
1.85 \\
1.83 \\
1.81 \\
1.79 \\
1.76 \\
1.74\end{array}$ & $\begin{array}{l}1.81 \\
1.80 \\
1.78 \\
1.77 \\
1.75 \\
1.73 \\
1.71 \\
1.69 \\
1.67 \\
1.64\end{array}$ & $\begin{array}{l}1.72 \\
1.71 \\
1.70 \\
1.68 \\
1.66 \\
1.65 \\
1.63 \\
1.61 \\
1.58 \\
1.56\end{array}$ & $\begin{array}{l}1.65 \\
1.63 \\
1.62 \\
1.60 \\
1.59 \\
1.57 \\
1.55 \\
1.53 \\
1.51 \\
1.49\end{array}$ & $\begin{array}{l}1.58 \\
1.56 \\
1.55 \\
1.54 \\
1.52 \\
1.51 \\
1.49 \\
1.47 \\
1.45 \\
1.43\end{array}$ & $\begin{array}{l}1.52 \\
1.50 \\
1.49 \\
1.48 \\
1.46 \\
1.45 \\
1.43 \\
1.41 \\
1.39 \\
1.37\end{array}$ & $\begin{array}{l}1.46 \\
1.45 \\
1.44 \\
1.43 \\
1.41 \\
1.40 \\
1.38 \\
1.36 \\
1.34 \\
1.33\end{array}$ & $\begin{array}{l}1.41 \\
1.40 \\
1.39 \\
1.38 \\
1.36 \\
1.35 \\
1.33 \\
1.32 \\
1.30 \\
1.28\end{array}$ & $\begin{array}{l}1.37 \\
1.36 \\
1.35 \\
1.34 \\
1.32 \\
1.31 \\
1.29 \\
1.28 \\
1.26 \\
1.24\end{array}$ \\
\hline $\begin{array}{l}.71 \\
.72 \\
.73 \\
.74 \\
.75 \\
.76 \\
.77 \\
.78 \\
.79 \\
.89\end{array}$ & $\begin{array}{l}1.82 \\
1.79 \\
1.76 \\
1.73 \\
1.69 \\
1.65 \\
1.61 \\
1.58 \\
1.53 \\
1.49\end{array}$ & $\begin{array}{l}1.71 \\
1.68 \\
1.65 \\
1.62 \\
1.59 \\
1.55 \\
1.52 \\
1.48 \\
1.44 \\
1.40\end{array}$ & $\begin{array}{l}1.62 \\
1.59 \\
1.56 \\
1.53 \\
1.50 \\
1.47 \\
1.43 \\
1.40 \\
1.36 \\
1.32\end{array}$ & $\begin{array}{l}1.54 \\
1.51 \\
1.49 \\
1.46 \\
1.43 \\
1.40 \\
1.36 \\
1.33 \\
1.29 \\
1.26\end{array}$ & $\begin{array}{l}1.47 \\
1.44 \\
1.42 \\
1.39 \\
1.36 \\
1.33 \\
1.30 \\
1.27 \\
1.24 \\
1.20\end{array}$ & $\begin{array}{l}1.41 \\
1.38 \\
1.36 \\
1.33 \\
1.31 \\
1.28 \\
1.25 \\
1.22 \\
1.18 \\
1.15\end{array}$ & $\begin{array}{l}1.35 \\
1.33 \\
1.31 \\
1.28 \\
1.26 \\
1.23 \\
1.29 \\
1.17 \\
1.14 \\
1.11\end{array}$ & $\begin{array}{l}1.30 \\
1.28 \\
1.26 \\
1.24 \\
1.21 \\
1.18 \\
1.16 \\
1.13 \\
1.10 \\
1.07\end{array}$ & $\begin{array}{l}1.26 \\
1.24 \\
1.22 \\
1.20 \\
1.17 \\
1.15 \\
1.12 \\
1.09 \\
1.06 \\
1.03\end{array}$ & $\begin{array}{l}1.22 \\
1.20 \\
1.18 \\
1.16 \\
1.14 \\
1.11 \\
1.08 \\
1.06 \\
1.03 \\
1.00\end{array}$ \\
\hline $\begin{array}{l}.81 \\
.82 \\
.83 \\
.84 \\
.85 \\
.86 \\
.87 \\
.88 \\
.89 \\
.90\end{array}$ & $\begin{array}{l}1.44 \\
1.40 \\
1.35 \\
1.29 \\
1.24 \\
1.18 \\
1.13 \\
1.06 \\
1.00 \\
0.93\end{array}$ & $\begin{array}{l}1.36 \\
1.31 \\
1.26 \\
1.22 \\
1.16 \\
1.11 \\
1.06 \\
1.00 \\
0.94 \\
0.88\end{array}$ & $\begin{array}{l}1.28 \\
1.24 \\
1.20 \\
1.15 \\
1.10 \\
1.05 \\
1.00 \\
0.95 \\
0.89 \\
0.83\end{array}$ & $\begin{array}{l}1.22 \\
1.18 \\
1.14 \\
1.09 \\
1.05 \\
1.09 \\
0.95 \\
0.94 \\
0.84 \\
0.79\end{array}$ & $\begin{array}{l}1.16 \\
1.13 \\
1.09 \\
1.04 \\
1.00 \\
0.95 \\
0.91 \\
0.86 \\
0.81 \\
0.75\end{array}$ & $\begin{array}{l}1.12 \\
1.08 \\
1.04 \\
1.00 \\
0.96 \\
0.91 \\
0.87 \\
0.82 \\
0.77 \\
0.72\end{array}$ & $\begin{array}{l}1.07 \\
1.04 \\
1.00 \\
0.96 \\
0.92 \\
0.88 \\
0.84 \\
0.79 \\
0.74 \\
0.69\end{array}$ & $\begin{array}{l}1.03 \\
1.010 \\
0.96 \\
0.93 \\
0.89 \\
0.85 \\
0.81 \\
0.76 \\
0.72 \\
0.67\end{array}$ & $\begin{array}{l}1.00 \\
0.97 \\
0.93 \\
0.90 \\
0.86 \\
0.82 \\
0.78 \\
0.74 \\
0.69 \\
0.65\end{array}$ & $\begin{array}{l}0.97 \\
0.94 \\
0.90 \\
0.87 \\
0.83 \\
0.80 \\
0.76 \\
0.71 \\
0.67 \\
0.63\end{array}$ \\
\hline $\begin{array}{r}.91 \\
.92 \\
.93 \\
.94 \\
.95 \\
.96 \\
.97 \\
.98 \\
.99\end{array}$ & $\begin{array}{l}0.86 \\
0.79 \\
0.71 \\
0.63 \\
0.55 \\
0.46 \\
0.36 \\
0.26 \\
0.14\end{array}$ & $\begin{array}{l}0.81 \\
0.74 \\
0.67 \\
0.60 \\
0.52 \\
0.43 \\
0.34 \\
0.24 \\
0.13\end{array}$ & $\begin{array}{l}0.77 \\
0.70 \\
0.63 \\
0.56 \\
0.49 \\
0.41 \\
0.32 \\
0.23 \\
0.13\end{array}$ & $\begin{array}{l}0.73 \\
0.67 \\
0.60 \\
0.54 \\
0.46 \\
0.39 \\
0.31 \\
0.22 \\
0.12\end{array}$ & $\begin{array}{l}0.70 \\
0.64 \\
0.58 \\
0.51 \\
0.44 \\
0.37 \\
0.29 \\
0.21 \\
0.11\end{array}$ & $\begin{array}{l}0.67 \\
0.61 \\
0.55 \\
0.49 \\
0.42 \\
0.35 \\
0.28 \\
0.20 \\
0.11\end{array}$ & $\begin{array}{l}0.64 \\
0.59 \\
0.53 \\
0.47 \\
0.41 \\
0.34 \\
0.27 \\
0.19 \\
0.11\end{array}$ & $\begin{array}{l}0.62 \\
0.57 \\
0.51 \\
0.45 \\
0.39 \\
0.33 \\
0.26 \\
0.18 \\
0.10\end{array}$ & $\begin{array}{l}0.61 \\
0.55 \\
0.49 \\
0.44 \\
0.38 \\
0.32 \\
0.25 \\
0.18 \\
0.10\end{array}$ & $\begin{array}{l}0.58 \\
0.53 \\
0.48 \\
0.43 \\
0.37 \\
0.31 \\
0.24 \\
0.17 \\
0.10\end{array}$ \\
\hline
\end{tabular}


Table 1 Continued

\begin{tabular}{|c|c|c|c|c|c|c|c|c|c|c|}
\hline $\begin{array}{c}\text { Hits } \\
{[p(\text { yes } \mid \text { sn })]}\end{array}$ & & & & Fal & Alarms & $e s \mid n)]$ & & & & \\
\hline & .21 & .22 & .23 & .24 & .25 & .26 & .27 & .28 & .29 & .30 \\
\hline .01 & 0.09 & 0.09 & 0.09 & 0.09 & 0.08 & 0.08 & ט. 08 & 0.08 & 0.08 & 0.08 \\
\hline .02 & 0.17 & 0.16 & 0.16 & 0.16 & 0.15 & 0.15 & 0.15 & 0.14 & 0.14 & 0.14 \\
\hline .03 & 0.24 & 0.23 & 0.22 & 0.22 & 0.21 & 0.21 & 0.21 & 0.20 & 0.20 & 0.20 \\
\hline .04 & 0.30 & 0.29 & 0.28 & 0.28 & 0.27 & 0.27 & 0.26 & 0.26 & 0.25 & 0.25 \\
\hline .05 & 0.36 & 0.35 & 0.34 & 0.33 & 0.32 & ט. 32 & 0.31 & 0.31 & 0.30 & 0.30 \\
\hline .06 & 0.41 & 0.40 & 0.39 & 0.38 & 0.37 & 0.37 & 0.36 & 0.35 & 0.35 & 0.34 \\
\hline .07 & 0.47 & 0.45 & 0.44 & 0.43 & 0.42 & 0.41 & 0.41 & 0.40 & 0.39 & 0.39 \\
\hline .08 & 0.52 & 0.50 & 0.49 & 0.48 & 0.47 & 0.46 & 0.45 & 0.44 & 0.43 & 0.43 \\
\hline .09 & 0.56 & 0.55 & 0.54 & 0.52 & 0.51 & 0.50 & 0.49 & 0.48 & 0.47 & 0.47 \\
\hline .10 & 0.61 & 0.59 & $D .58$ & 0.56 & 0.55 & 0.54 & 0.53 & 0.52 & 0.51 & 0.50 \\
\hline .11 & 0.65 & 0.63 & 0.62 & 0.60 & 0.59 & 0.58 & 0.57 & 0.56 & 0.55 & 0.54 \\
\hline .12 & 0.69 & 0.68 & 0.66 & 0.64 & 0.63 & 0.62 & 0.61 & 0.59 & D. 58 & 0.58 \\
\hline .13 & 0.73 & 0.71 & 0.70 & 0.68 & 0.67 & 0.65 & 0.64 & 0.63 & 0.62 & 0.61 \\
\hline .14 & 0.77 & 0.75 & 0.73 & 0.72 & 0.70 & 0.69 & 0.67 & 0.66 & 0.65 & 0.64 \\
\hline .15 & 0.81 & 0.79 & 0.77 & 0.75 & 0.73 & 0.72 & 0.70 & 0.69 & 0.68 & 0.67 \\
\hline .16 & 0.84 & 0.82 & 0.80 & 0.78 & 0.77 & 0.75 & 0.74 & 0.72 & 0.71 & 0.70 \\
\hline .17 & 0.88 & 0.85 & 0.83 & 0.81 & 0.80 & 0.78 & 0.77 & 0.75 & 0.74 & 0.73 \\
\hline .18 & 0.91 & D. 89 & 0.86 & 0.84 & 0.83 & 0.81 & 0.79 & 0.78 & 0.77 & 0.75 \\
\hline .19 & 0.94 & 0.92 & 0.89 & 0.87 & 0.85 & 0.84 & 0.82 & 0.81 & 0.79 & 0.78 \\
\hline .20 & 0.97 & 0.95 & 0.92 & 0.90 & 0.88 & 0.86 & 0.85 & 0.83 & 0.82 & 0.81 \\
\hline .21 & 1.00 & 0.97 & 0.95 & 0.93 & 0.91 & 0.89 & 0.87 & 0.86 & 0.84 & 0.83 \\
\hline .22 & 1.03 & 1.00 & 0.98 & 0.95 & 0.93 & 0.91 & 0.90 & 0.88 & 0.87 & 0.85 \\
\hline .23 & 1.05 & 1.03 & 1.00 & 0.98 & 0.96 & 0.94 & 10.92 & 0.90 & 0.89 & 0.87 \\
\hline .24 & 1.08 & 1.05 & 1.02 & 1.00 & 0.98 & 0.96 & 0.94 & 0.92 & 0.91 & 0.89 \\
\hline .25 & 1.10 & 1.07 & 1.05 & 1.02 & 1.106 & 0.98 & 0.96 & 0.94 & 0.93 & 0.91 \\
\hline .26 & 1.13 & 1.10 & 1.07 & 1.04 & 1.02 & 1.00 & 0.98 & 0.96 & 0.95 & 0.93 \\
\hline .27 & 1.15 & 1.12 & 1.09 & 1.06 & 1.04 & 1.02 & 1.00 & 0.98 & 0.97 & 0.95 \\
\hline .28 & 1.17 & 1.14 & 1.11 & 1.08 & 1.06 & 1.04 & 1.02 & 1.00 & 0.98 & 0.97 \\
\hline .29 & 1.19 & 1.16 & 1.13 & 1.10 & 1.08 & 1.05 & 1.03 & 1.02 & 1.00 & 0.98 \\
\hline .30 & 1.21 & 1.17 & 1.15 & 1.12 & 1.09 & 1.07 & 1.05 & 1.03 & 1.02 & 1.00 \\
\hline .31 & 1.22 & 1.19 & 1.16 & 1.13 & 1.11 & 1.09 & 1.07 & 1.05 & 1.03 & 1.01 \\
\hline .32 & 1.24 & 1.21 & 1.18 & 1.15 & 1.13 & 1.10 & 1.08 & 1.06 & 1.105 & 1.03 \\
\hline .33 & 1.26 & 1.22 & 1.19 & 1.16 & 1.14 & 1.12 & 1.09 & 1.08 & 1.06 & 1.84 \\
\hline .34 & 1.27 & 1.24 & 1.21 & 1.18 & 1.15 & 1.13 & 1.11 & 1.09 & 1.07 & 1.05 \\
\hline .35 & 1.29 & 1.25 & 1.22 & 1.19 & 1.17 & 1.14 & 1.12 & 1.10 & 1.08 & 1.07 \\
\hline .36 & 1.30 & 1.26 & 1.23 & 1.20 & 1.18 & 1.15 & 1.13 & 1.11 & 1.09 & 1.08 \\
\hline .37 & 1.31 & 1.28 & 1.24 & 1.21 & 1.19 & 1.16 & 1.14 & 1.12 & 1.10 & 1.09 \\
\hline .38 & 1.32 & 1.29 & 1.25 & 1.22 & 1.20 & 1.17 & 1.15 & 1.13 & 1.11 & 1.09 \\
\hline .39 & 1.33 & 1.30 & 1.26 & 1.23 & 1.21 & 1.18 & 1.16 & 1.14 & 1.12 & 1.10 \\
\hline .40 & 1.34 & 1.30 & 1.27 & 1.24 & 1.22 & 1.19 & 1.17 & 1.15 & 1.13 & 1.11 \\
\hline .41 & 1.35 & 1.31 & 1.28 & 1.25 & 1.22 & 1.20 & 1.18 & 1.15 & 1.14 & 1.12 \\
\hline .42 & 1.36 & 1.32 & 1.29 & 1.26 & 1.23 & 1.20 & 1.18 & 1.16 & 1.14 & 1.12 \\
\hline .43 & 1.36 & 1.33 & 1.29 & 1.26 & 1.24 & 1.21 & 1.19 & 1.17 & 1.15 & 1.13 \\
\hline .44 & 1.37 & 1.33 & 1.30 & 1.27 & 1.24 & 1.22 & 1.19 & 1.17 & 1.15 & 1.13 \\
\hline .45 & 1.37 & 1.34 & 1.30 & 1.27 & 1.25 & 1.22 & 1.20 & 1.18 & 1.16 & 1.14 \\
\hline .46 & 1.38 & 1.34 & 1.31 & 1.28 & 1.25 & 1.22 & 1.20 & 1.18 & 1.16 & 1.14 \\
\hline .47 & 1.38 & 1.34 & 1.31 & 1.28 & 1.25 & 1.23 & 1.20 & 1.18 & 1.16 & 1.14 \\
\hline .48 & 1.38 & 1.35 & 1.31 & 1.28 & 1.25 & 1.23 & 1.20 & 1.18 & 1.16 & 1.15 \\
\hline .49 & 1.38 & 1.35 & 1.31 & 1.28 & 1.25 & 1.23 & 1.21 & 1.18 & 1.17 & 1.15 \\
\hline .50 & 1.38 & 1.35 & 1.31 & 1.28 & 1.26 & 1.23 & 1.21 & 1.19 & 1.17 & 1.15 \\
\hline
\end{tabular}


Table 1 Continued

\begin{tabular}{|c|c|c|c|c|c|c|c|c|c|c|}
\hline $\begin{array}{c}\text { Hits } \\
{[\mathrm{p}(\text { yes } \mid \mathrm{sn})]}\end{array}$ & & & & Fal & Alarms II & & & & & \\
\hline & .21 & .22 & .23 & .24 & .25 & .26 & .27 & .28 & .29 & .30 \\
\hline & $\begin{array}{r}0 z \\
.79\end{array}$ & $\begin{array}{l}\text { or } \\
.78\end{array}$ & $\begin{array}{r}0 . \\
.77\end{array}$ & $\begin{array}{r}0: \\
.76\end{array}$ & $\begin{array}{r}0 . \\
.75\end{array}$ & $\begin{array}{r}0= \\
.74\end{array}$ & $\begin{array}{l}.8 \\
.73\end{array}$ & $\begin{array}{l}02 \\
.72\end{array}$ & $\begin{array}{l}\text { or } \\
.71\end{array}$ & $\begin{array}{l}08 \\
.70\end{array}$ \\
\hline .51 & 1.38 & 1.35 & 1.31 & 1.28 & 1.25 & 1.23 & 1.21 & 1.18 & 1.17 & 1.15 \\
\hline .52 & 1.38 & 1.35 & 1.31 & 1.28 & 1.25 & 1.23 & 1.20 & 1.18 & 1.16 & 1.15 \\
\hline .53 & 1.38 & 1.34 & 1.31 & 1.28 & 1.25 & 1.23 & 1.20 & 1.18 & 1.16 & 1.14 \\
\hline .54 & 1.38 & 1.34 & 1.31 & 1.28 & 1.25 & 1.22 & 1.20 & 1.18 & 1.16 & 1.14 \\
\hline .55 & 1.37 & 1.34 & 1.30 & 1.27 & 1.25 & 1.22 & 1.20 & 1.18 & 1.16 & 1.14 \\
\hline .56 & 1.37 & 1.33 & 1.30 & 1.27 & 1.24 & 1.22 & 1.19 & 1.17 & 1.15 & 1.13 \\
\hline .57 & 1.36 & 1.33 & 1.29 & 1.26 & 1.24 & 1.21 & 1.19 & 1.17 & 1.15 & 1.13 \\
\hline .58 & 1.36 & 1.32 & 1.29 & 1.26 & 1.23 & 1.20 & 1.18 & 1.16 & 1.14 & 1.12 \\
\hline .59 & 1.35 & 1.31 & 1.28 & 1.25 & 1.22 & 1.20 & 1.18 & 1.15 & 1.14 & 1.12 \\
\hline .60 & 1.34 & 1.30 & 1.27 & 1.24 & 1.22 & 1.19 & 1.17 & 1.15 & 1.13 & 1.11 \\
\hline .61 & 1.33 & 1.30 & 1.26 & 1.23 & 1.21 & 1.18 & 1.16 & 1.14 & 1.12 & 1.10 \\
\hline .62 & 1.32 & 1.29 & 1.25 & 1.22 & 1.20 & 1.17 & 1.15 & 1.13 & 1.11 & 1.09 \\
\hline .63 & 1.31 & 1.28 & 1.24 & 1.21 & 1.19 & 1.16 & 1.14 & 1.12 & 1.10 & 1.09 \\
\hline .64 & 1.30 & 1.26 & 1.23 & 1.20 & 1.18 & 1.15 & 1.13 & 1.11 & 1.09 & 1.08 \\
\hline .65 & 1.29 & 1.25 & 1.22 & 1.19 & 1.17 & 1.14 & 1.12 & 1.10 & 1.08 & 1.07 \\
\hline .66 & 1.27 & 1.24 & 1.21 & 1.18 & 1.15 & 1.13 & 1.11 & 1.09 & 1.07 & 1.05 \\
\hline .67 & 1.26 & 1.22 & 1.19 & 1.16 & 1.14 & 1.12 & 1.09 & 1.08 & 1.06 & 1.04 \\
\hline .68 & 1.24 & 1.21 & 1.18 & 1.15 & 1.13 & 1.10 & 1.08 & 1.06 & 1.05 & 1.03 \\
\hline .69 & 1.22 & 1.19 & 1.16 & 1.13 & 1.11 & 1.69 & 1.07 & 1.05 & 1.03 & 1.01 \\
\hline .70 & 1.21 & 1.17 & 1.15 & 1.12 & 1.89 & 1.07 & 1.05 & 1.03 & 1.02 & 1.00 \\
\hline .71 & 1.19 & 1.16 & 1.13 & 1.10 & 1.08 & 1.65 & 1.03 & 1.02 & 1.60 & 0.98 \\
\hline .72 & 1.17 & 1.14 & 1.11 & 1.08 & 1.06 & 1.04 & 1.02 & 1.00 & 0.5 & 0.97 \\
\hline .73 & 1.15 & 1.12 & 1.09 & 1.06 & 1.04 & 1.02 & 1.60 & 0.98 & 10.97 & 0.95 \\
\hline .74 & 1.13 & 1.10 & 1.07 & 1.04 & 1.62 & 1.06 & 0.98 & 4.96 & 0.95 & 0.93 \\
\hline .75 & 1.10 & 1.07 & 1.05 & 1.02 & 1.00 & 0.98 & 0.96 & 0.94 & 9.93 & 0.91 \\
\hline .76 & 1.08 & 1.05 & 1.02 & 1.00 & 0.98 & 0.96 & 0.94 & 0.92 & 0.91 & 0.89 \\
\hline .77 & 1.05 & 1.03 & 1.00 & 0.98 & 0.96 & 0.94 & 10.92 & 0.90 & 0.89 & 0.87 \\
\hline .78 & 1.03 & 1.00 & 0.98 & 0.95 & 0.93 & 0.91 & 0.90 & 0.88 & 0.87 & 0.85 \\
\hline .79 & 1.00 & 0.97 & 9.95 & 0.93 & 0.91 & 3.89 & 0.87 & 1. 86 & 0.84 & 0.83 \\
\hline .80 & 0.97 & 0.95 & 0.92 & 0.90 & 0.88 & 0.86 & 0.85 & 0.83 & 0.82 & 0.81 \\
\hline .81 & 6.94 & 0.92 & 0.89 & 0.87 & 0.85 & 0.84 & 0.82 & 0.81 & 0.79 & 0.78 \\
\hline .82 & 0.91 & 0.89 & 0.86 & 0.84 & 0.83 & 0.81 & 0.79 & 0.78 & 0.77 & 0.75 \\
\hline .83 & 0.88 & 0.85 & 0.83 & 0.81 & 0.80 & 0.78 & 0.77 & 0.75 & 0.74 & 0.73 \\
\hline .84 & 0.84 & 0.82 & 0.80 & 0.78 & 0.77 & 0.75 & 0.74 & 0.72 & 0.71 & 0.70 \\
\hline .85 & 0.81 & 0.79 & 6.77 & 0.75 & 0.73 & 0.72 & 0.70 & 0.69 & 0.68 & 0.67 \\
\hline .86 & 0.77 & 8.75 & 0.73 & 0.72 & 0.70 & 0.69 & v. 67 & 0.66 & 0.65 & 0.64 \\
\hline .87 & 0.73 & 0.71 & 0.70 & 0.68 & 0.67 & 0.65 & 0.64 & 0.63 & 0.62 & 0.61 \\
\hline .88 & 0.69 & 0.68 & 0.66 & 0.64 & 0.63 & 0.62 & 0.61 & 0.59 & 0.58 & 0.58 \\
\hline .89 & 0.65 & 0.63 & 0.62 & 0.60 & 0.59 & 0.58 & 0.57 & 4.56 & 0.55 & 0.54 \\
\hline .90 & 0.61 & 0.59 & 0.58 & 0.56 & 0.55 & 0.54 & 0.53 & 0.52 & 0.51 & 0.56 \\
\hline .91 & 0.56 & 0.55 & 0.54 & 0.52 & 0.51 & 0.50 & 0.49 & 0.4 & 0.47 & 0.47 \\
\hline .92 & 0.52 & 0.50 & 0.49 & 0.48 & 0.47 & 0.46 & $\mathfrak{0 . 4 5}$ & 0.44 & 0.43 & 0.43 \\
\hline .93 & 0.47 & 0.45 & 0.44 & 0.43 & 6.42 & 0.41 & 0.41 & 0.46 & 0.39 & 0.39 \\
\hline .94 & 0.41 & 0.40 & 0.39 & 10.38 & 0.37 & 9.37 & 0.36 & 0.35 & 0.35 & 0.34 \\
\hline .95 & 0.36 & 0.35 & 0.34 & 0.33 & 0.32 & 0.32 & 0.31 & 0.31 & 0.30 & 0.30 \\
\hline .96 & 0.310 & 0.29 & 0.28 & 0.28 & 0.27 & 0.27 & 0.26 & 0.26 & 0.25 & 0.25 \\
\hline .97 & 0.24 & 9.23 & 0.22 & 0.22 & 0.21 & 0.21 & 10.21 & 8.20 & 0.20 & 0.29 \\
\hline .98 & 0.17 & 0.16 & 0.16 & 0.16 & 0.15 & $\mathfrak{0} .15$ & 9.15 & 0.14 & 0.14 & 0.14 \\
\hline • 99 & 0.09 & 0.09 & 0.09 & 0.09 & 0.08 & 0.08 & 0.68 & 0.08 & 0.08 & 0.08 \\
\hline
\end{tabular}


Table 1 Continued

Hits

[p(yes $\mid \mathrm{sn})]$
False Alarms [p(yes $\mid \mathbf{n})]$

\begin{tabular}{|c|c|c|c|c|c|c|c|c|c|c|}
\hline & .31 & .32 & .33 & .34 & .35 & .36 & .37 & .38 & .39 & .40 \\
\hline .01 & 0.08 & 0.07 & 0.07 & 0.07 & 0.07 & 0.67 & 0.07 & 0.07 & 0.07 & 10.07 \\
\hline .02 & 0.14 & 0.14 & 0.13 & 0.13 & 0.13 & 0.13 & 0.13 & 0.13 & 0.13 & 0.13 \\
\hline .03 & 0.19 & 0.19 & 0.19 & 0.19 & 0.18 & 0.18 & 0.18 & 0.18 & 0.18 & 0.18 \\
\hline .04 & 0.24 & 0.24 & 0.24 & 0.23 & 0.23 & 0.23 & 0.23 & 0.23 & 0.22 & 0.22 \\
\hline .05 & 0.29 & 0.29 & 0.29 & 0.28 & 0.28 & 0.28 & 0.27 & 0.27 & ט. 27 & 0.27 \\
\hline .06 & 0.34 & 0.33 & 0.33 & 0.33 & 1. 32 & 0.32 & 0.32 & 0.31 & 0.31 & 0.31 \\
\hline .07 & 0.38 & 0.38 & 0.37 & 0.37 & 0.36 & 0.36 & 0.36 & 0.35 & 0.35 & 0.35 \\
\hline .08 & 0.42 & 0.42 & 0.41 & 0.41 & 0.40 & 0.40 & 0.39 & 0.39 & 0.39 & 0.38 \\
\hline .09 & 0.46 & 0.45 & 0.45 & 0.44 & 0.44 & 0.43 & 0.43 & 0.43 & 0.42 & 0.42 \\
\hline .10 & 0.50 & 0.49 & 0.48 & 0.48 & 0.47 & 0.47 & 0.46 & 0.46 & 0.46 & 0.45 \\
\hline .11 & 0.53 & 0.53 & 0.52 & 0.51 & 0.51 & 0.50 & 0.50 & 0.49 & 0.49 & 0.49 \\
\hline .12 & 0.57 & 0.56 & 0.55 & 0.55 & 0.54 & 0.53 & 0.53 & 0.53 & ט. 52 & 0.52 \\
\hline .13 & 0.60 & 0.59 & 0.58 & 0.58 & 0.57 & 0.57 & 0.56 & 0.56 & 0.55 & 0.55 \\
\hline .14 & 0.63 & 0.62 & 0.61 & 0.61 & 0.60 & 0.60 & 0.59 & 0.58 & 0.58 & 0.58 \\
\hline .15 & 0.66 & 0.65 & 0.64 & 0.64 & 0.63 & 0.62 & 0.62 & 0.61 & 0.61 & 0.60 \\
\hline .16 & 0.69 & 0.68 & 0.67 & 0.66 & 0.66 & 0.65 & 0.64 & 0.64 & 0.63 & 0.63 \\
\hline .17 & 0.72 & 0.71 & 0.70 & 0.69 & 0.68 & 0.68 & 0.67 & 0.66 & 0.66 & 0.65 \\
\hline .18 & 0.74 & 0.73 & 0.72 & 0.72 & 0.71 & 0.70 & 0.69 & 0.69 & 0.68 & 0.68 \\
\hline .19 & 0.77 & 0. 76 & 0.75 & 0.74 & 0.73 & 0.73 & 0.72 & 0.71 & 0.71 & 0.70 \\
\hline .280 & 0.79 & 0.78 & 0.77 & 0.76 & 0.76 & 0.75 & 0.74 & 0.74 & 0.73 & 0.72 \\
\hline .21 & 0.82 & 0.81 & 0.80 & 0.79 & 0.78 & 0.77 & 0.76 & 0.76 & 0.75 & 0.75 \\
\hline .22 & 0.84 & 0.83 & 6.82 & 0.81 & 0.810 & 0.79 & 0.78 & 0.78 & 0.77 & 0.77 \\
\hline .23 & 0.86 & 0.85 & 0.84 & 0.83 & 0.82 & 0.81 & 0.80 & 0.80 & 0.79 & 0.79 \\
\hline .24 & 0.88 & 0.87 & 0.86 & 0.85 & 0.84 & 0.83 & 0.82 & 0.82 & 0.81 & 0.80 \\
\hline .25 & 0.90 & 0.89 & 0.88 & $\emptyset .87$ & 0.86 & 0.85 & 0.84 & 0.83 & 0.83 & 0.82 \\
\hline .26 & 0.92 & 0.91 & 0.90 & 0.89 & 9.88 & 0.87 & 0.86 & 0.85 & 0.85 & 0.84 \\
\hline .27 & 0.94 & 0.92 & 0.91 & 4.90 & 0.89 & Ø. 88 & 0.88 & 0.87 & 0.86 & 0.86 \\
\hline .28 & 0.95 & 0.94 & 0.93 & 0.92 & 0.91 & 0.90 & 0.89 & 0.88 & 0.88 & 0.87 \\
\hline .29 & 0.97 & 0.96 & 0.95 & 0.93 & 0.92 & 0.91 & 0.91 & 0.90 & 0.89 & 0.89 \\
\hline . 30 & 0.99 & 0.97 & 0.96 & 0.95 & 0.94 & 0.93 & 0.92 & 0.91 & 0.91 & 0.90 \\
\hline .31 & 1.00 & 0.99 & 0.97 & 0.96 & 0.95 & 0.94 & 0.93 & 0.93 & 0.92 & 0.91 \\
\hline .32 & 1.01 & 1.00 & 0.99 & 0.98 & 0.97 & 10.96 & 0.95 & $\emptyset .94$ & 0.93 & 0.93 \\
\hline .33 & 1.03 & 1.01 & 1.06 & 0.99 & 0.98 & 0.97 & 0.96 & 0.95 & 0.94 & 0.94 \\
\hline .34 & 1.04 & 1.62 & 1.61 & 1.00 & 0.99 & $\$ .98$ & 0.97 & 0.96 & 0.95 & 0.95 \\
\hline .35 & 1.05 & 1.04 & 1.02 & 1.01 & 1.06 & 0.99 & 0.98 & 0.97 & 0.97 & 0.96 \\
\hline . 36 & 1.06 & 1.05 & 1.03 & 1.02 & 1.01 & 1.06 & 10.99 & 0.98 & 0.97 & 0.97 \\
\hline .37 & 1.07 & 1.06 & 1.04 & 1.03 & 1.02 & 1.01 & 1.00 & 0.99 & 0.98 & 0.98 \\
\hline . 38 & 1.08 & 1.06 & 1.05 & 1.04 & 1.03 & 1.02 & 1.01 & 1.00 & 0.99 & 0.99 \\
\hline .39 & 1.09 & 1.07 & 1.06 & 1.65 & 1.04 & 1.03 & 1.02 & 1.01 & 1.00 & 0.99 \\
\hline .40 & 1.10 & 1.08 & 1.07 & 1.05 & 1.04 & 1.03 & 1.02 & 1.01 & 1.01 & 1.00 \\
\hline .41 & 1.10 & 1.09 & 1.07 & 1.66 & 1.05 & 1.04 & 1.03 & 1.02 & 1.01 & 1.01 \\
\hline .42 & 1.11 & 1.09 & 1.68 & 1.07 & 1.06 & 1.04 & 1.04 & 1.03 & 1.02 & 1.01 \\
\hline .43 & 1.11 & 1.10 & 1.08 & 1.07 & 1.06 & 1.05 & 1.04 & 1.03 & 1.02 & 1.02 \\
\hline . 44 & 1.12 & 1.10 & 1.69 & 1.08 & 1.06 & 1.05 & 1.04 & 1.04 & 1.03 & 1.02 \\
\hline .45 & 1.12 & 1.11 & 1.09 & 1.08 & 1.07 & 1.06 & 1.05 & 1.04 & 1.03 & 1.02 \\
\hline .46 & 1.13 & 1.11 & 1.10 & 1.08 & 1.07 & 1.66 & 1.85 & 1.04 & 1.03 & 1.03 \\
\hline .47 & 1.13 & 1.11 & 1.10 & 1.09 & 1.07 & 1.06 & 1.05 & 1.04 & 1.04 & 1.03 \\
\hline .48 & 1.13 & 1.11 & 1.16 & 1.09 & 1.08 & 1.06 & 1.06 & 1.05 & 1.04 & 1.03 \\
\hline .49 & 1.13 & 1.12 & 1.10 & 1.09 & 1.08 & 1.67 & 1.06 & 1.05 & 1.64 & 1.03 \\
\hline .50 & 1.13 & 1.12 & 1.10 & 1.99 & 1.08 & 1.07 & 1.06 & 1.05 & 1.04 & 1.03 \\
\hline
\end{tabular}


Table 1 Continued

\begin{tabular}{|c|c|c|c|c|c|c|c|c|c|c|}
\hline \multirow[t]{3}{*}{$\begin{array}{c}\begin{array}{c}\text { Hits } \\
\text { [p(yes } \mid \text { sn })]\end{array} \\
\end{array}$} & \multicolumn{10}{|c|}{ False Alarms [p(yes $\mid n)]$} \\
\hline & .31 & .32 & .33 & .34 & .35 & .36 & .37 & .38 & .39 & .40 \\
\hline & $\begin{array}{l}0: \\
.69\end{array}$ & $\begin{array}{r}0: \\
.68\end{array}$ & $\begin{array}{l}02 \\
.67\end{array}$ & $\begin{array}{l}02 \\
.66\end{array}$ & $\begin{array}{r}02 \\
.65\end{array}$ & $\begin{array}{r}0: \\
.64\end{array}$ & $\begin{array}{r}0 r \\
.63\end{array}$ & $\begin{array}{l}0 x \\
.62\end{array}$ & $\begin{array}{r}0 r \\
.61\end{array}$ & $\begin{array}{l}0: \\
.600\end{array}$ \\
\hline .51 & 1.13 & 1.12 & 1.10 & 1.09 & 1.08 & 1.07 & 1.06 & 1.05 & 1.04 & 1.03 \\
\hline .52 & 1.13 & 1.11 & 1.10 & 1.69 & 1.08 & 1.06 & 1.06 & 1.05 & 1.04 & 1.03 \\
\hline .53 & 1.13 & 1.11 & 1.10 & 1.09 & 1.07 & 1.06 & 1.05 & 1.04 & 1.04 & 1.03 \\
\hline .54 & 1.13 & 1.11 & 1.10 & 1.08 & 1.07 & 1.06 & 1.05 & 1.04 & 1.03 & 1.03 \\
\hline .55 & 1.12 & 1.11 & 1.09 & 1.08 & 1.07 & 1.06 & 1.05 & 1.04 & 1.03 & 1.02 \\
\hline .56 & 1.12 & 1.10 & 1.09 & 1.08 & 1.06 & 1.05 & 1.04 & 1.04 & 1.03 & 1.02 \\
\hline .57 & 1.11 & 1.10 & 1.08 & 1.07 & 1.06 & 1.05 & 1.04 & 1.03 & 1.02 & 1.02 \\
\hline .58 & 1.11 & 1.09 & 1.08 & 1.07 & 1.06 & 1.04 & 1.04 & 1.03 & 1.02 & 1.01 \\
\hline .59 & 1.10 & 1.09 & 1.07 & 1.06 & 1.05 & 1.04 & 1.03 & 1.02 & 1.01 & 1.01 \\
\hline .60 & 1.10 & 1.08 & 1.07 & 1.05 & 1.04 & 1.03 & 1.02 & 1.01 & 1.01 & 1.00 \\
\hline .61 & 1.09 & 1.07 & 1.06 & 1.05 & 1.04 & 1.03 & 1.62 & 1.01 & 1.013 & 0.99 \\
\hline .62 & 1.08 & 1.06 & 1.05 & 1.04 & 1.03 & 1.02 & 1.01 & 1.00 & 0.99 & 0.99 \\
\hline .63 & 1.07 & 1.06 & 1.04 & 1.03 & 1.02 & 1.01 & 1.00 & 0.99 & 0.98 & 0.98 \\
\hline .64 & 1.06 & 1.05 & 1.03 & 1.02 & 1.01 & 1.60 & 0.99 & 0.98 & 0.97 & 0.97 \\
\hline .65 & 1.05 & 1.04 & 1.02 & 1.01 & 1.00 & 0.99 & 0.98 & 0.97 & 0.97 & 0.96 \\
\hline .66 & 1.04 & 1.02 & 1.01 & 1.00 & 0.99 & 0.98 & 0.97 & 0.96 & 0.95 & 0.95 \\
\hline .67 & 1.03 & 1.01 & 1.00 & 0.99 & 0.98 & 0.97 & D. 96 & 0.95 & 0.94 & 0.94 \\
\hline .68 & 1.01 & 1.00 & 0.99 & 0.98 & 0.97 & 0.96 & 0.95 & 0.94 & 0.93 & 0.93 \\
\hline .69 & 1.00 & 0.99 & 0.97 & 0.96 & 0.95 & 0.94 & 0.93 & 0.93 & 0.92 & 0.91 \\
\hline .70 & 0.99 & 0.97 & 0.96 & 0.95 & 0.94 & 0.93 & 0.92 & 0.91 & 0.91 & 0.90 \\
\hline .71 & 0.97 & 0.96 & 0.95 & 0.93 & 0.92 & 0.91 & 0.91 & 0.90 & 0.89 & 0.89 \\
\hline .72 & 1.95 & 0.94 & v.93 & 0.92 & 0.91 & 0.90 & 0.89 & 0.88 & 0.88 & 0.87 \\
\hline .73 & 0.94 & 0.92 & 0.91 & 0.90 & 6.89 & 0.88 & 0.88 & 0.87 & 0.86 & 0.86 \\
\hline .74 & 0.92 & 0.91 & 0.90 & 0.89 & 0.88 & 0.87 & 0.86 & 0.85 & 0.85 & 0.84 \\
\hline .75 & 0.90 & 0.89 & 0.88 & 0.87 & 0.86 & 0.85 & 0.84 & 0.83 & 0.83 & 0.82 \\
\hline .76 & 0.88 & 0.87 & 0.86 & 0.85 & 0.84 & 0.83 & 0.82 & 0.82 & 0.81 & 0.80 \\
\hline .77 & 0.86 & 0.85 & 0.84 & 0.83 & 0.82 & 0.81 & 0.80 & 0.80 & 0.79 & 0.79 \\
\hline .78 & 0.84 & 0.83 & 0.82 & 0.81 & 0.80 & 0.79 & 0.78 & 0.78 & 0.77 & 0.77 \\
\hline .79 & 0.82 & 0.81 & 0.80 & 0.79 & 0.78 & 0.77 & 0.76 & 0.76 & 0.75 & 0.75 \\
\hline .80 & 0.79 & 0.78 & 0.77 & 10.76 & 0.76 & 0.75 & 0.74 & 0.74 & 0.73 & 0.72 \\
\hline .81 & 0.77 & 0.76 & 0.75 & 0.74 & 0.73 & 0.73 & 0.72 & 0.71 & 0.71 & 0.70 \\
\hline .82 & 0.74 & 0.73 & 0.72 & 0.72 & 0.71 & 0.70 & 0.69 & 0.69 & 0.68 & 0.68 \\
\hline .83 & 0.72 & 0.71 & 0.70 & 0.69 & 0.68 & 0.68 & 0.67 & 0.66 & 0.66 & 0.65 \\
\hline .84 & 0.69 & 0.68 & 0.67 & 0.66 & 0.66 & 0.65 & 0.64 & 0.64 & 0.63 & 0.63 \\
\hline .85 & 0.66 & 0.65 & 0.64 & 0.64 & 0.63 & 0.62 & 0.62 & 0.61 & 0.61 & 0.60 \\
\hline .86 & 0.63 & 0.62 & 0.61 & 0.61 & 0.60 & 0.60 & 0.59 & 0.58 & 0.58 & 0.58 \\
\hline .87 & 0.60 & 0.59 & 0.58 & 0.58 & 0.57 & 0.57 & 0.56 & 0.56 & 0.55 & 0.55 \\
\hline .88 & 0.57 & 0.56 & 0.55 & 0.55 & 0.54 & 0.53 & 0.53 & 0.53 & 0.52 & 0.52 \\
\hline .89 & 0.53 & 0.53 & 0.52 & 0.51 & 0.51 & 0.50 & 0.50 & 0.49 & 0.49 & 0.49 \\
\hline .90 & 0.50 & 0.49 & 0.48 & 0.48 & 0.47 & 0.47 & 0.46 & 0.46 & 0.46 & 0.45 \\
\hline .91 & 0.46 & 0.45 & 0.45 & 3.44 & 0.44 & 0.43 & 10.43 & 0.43 & 0.42 & 0.42 \\
\hline .92 & 0.42 & 0.42 & 0.41 & 0.41 & 0.40 & 0.40 & 0.39 & 0.39 & 0.39 & 0.38 \\
\hline .93 & 0.38 & 0.38 & 0.37 & 0.37 & ע. 36 & 0.36 & 0.36 & 0.35 & 0.35 & 0.35 \\
\hline .94 & 0.34 & 0.33 & 0.33 & 0.33 & 0.32 & 0.32 & 0.32 & 0.31 & 0.31 & 0.31 \\
\hline .95 & 0.29 & 0.29 & 0.29 & 0.28 & 0.28 & 10.28 & 0.27 & 0.27 & 0.27 & 0.27 \\
\hline .96 & 0.24 & 0.24 & 0.24 & 0.23 & 0.23 & 0.23 & 0.23 & 0.23 & 0.22 & 0.22 \\
\hline .97 & 0.19 & 0.19 & 0.19 & 0.19 & 0.18 & 0.18 & 0.18 & 0.18 & 0.18 & 0.18 \\
\hline .98 & 0.14 & 0.14 & 0.13 & 0.13 & 0.13 & 0.13 & 0.13 & 0.13 & 0.13 & 0.13 \\
\hline .99 & 6.08 & 0.07 & 10.07 & 0.07 & 0.07 & 0.07 & 0.07 & 0.07 & 0.07 & 0.07 \\
\hline
\end{tabular}


Table 1 Continued

\begin{tabular}{|c|c|c|c|c|c|c|c|c|c|c|}
\hline $\begin{array}{c}\text { Hits } \\
[\mathrm{p}(\mathrm{yes}\rangle \mathrm{sn})]\end{array}$ & & & & & Alarms [ & es $\mid n)]$ & & & & \\
\hline & .41 & .42 & .43 & .44 & .45 & .46 & .47 & .48 & .49 & .50 \\
\hline .01 & 0.07 & 0.07 & 0.07 & 0.07 & 0.07 & 0.07 & 0.07 & 0.07 & 0.07 & 0.07 \\
\hline .02 & 0.12 & 0.12 & 0.12 & 0.12 & 0.12 & 0.12 & 0.12 & 0.12 & 0.12 & 0.12 \\
\hline .03 & 0.17 & 0.17 & 0.17 & 0.17 & 0.17 & 0.17 & 0.17 & 0.17 & 0.17 & 0.17 \\
\hline .04 & 0.22 & 0.22 & 0.22 & 0.22 & 0.22 & 0.22 & 0.22 & 0.22 & 0.22 & 0.22 \\
\hline .05 & 0.27 & 0.26 & 0.26 & 0.26 & 0.26 & 0.26 & 0.26 & 0.26 & 0.26 & 0.26 \\
\hline .06 & 0.31 & 0.30 & 0.30 & 0.30 & 0.30 & 0.30 & 0.30 & 0.30 & 0.30 & 0.30 \\
\hline .07 & 0.35 & 0.34 & 0.34 & 0.34 & 0.34 & 0.34 & 0.34 & 0.34 & 0.34 & 0.34 \\
\hline .08 & 0.38 & 0.38 & 0.38 & 0.38 & 0.38 & 0.37 & 0.37 & 0.37 & 0.37 & 0.37 \\
\hline .09 & 0.42 & 0.42 & 0.41 & 0.41 & 0.41 & 0.41 & 0.41 & 0.41 & 0.41 & 0.41 \\
\hline .10 & 0.45 & 0.45 & 0.45 & 0.44 & 0.44 & 0.44 & 0.44 & 0.44 & 0.44 & 0.44 \\
\hline .11 & 0.48 & 0.48 & 0.48 & 0.48 & 0.47 & 0.47 & 0.47 & 0.47 & 3.47 & 0.47 \\
\hline .12 & 0.51 & 0.51 & 0.51 & 0.51 & 0.51 & 0.50 & 0.50 & 0.50 & 0.50 & 0.50 \\
\hline .13 & 0.54 & 0.54 & 0.54 & 0.54 & 0.53 & 0.53 & 0.53 & 0.53 & 0.53 & 0.53 \\
\hline .14 & 0.57 & 0.57 & 0.57 & 0.56 & 0.56 & 0.56 & 0.56 & 0.56 & 0.56 & 0.56 \\
\hline .15 & 0.60 & 0.60 & 0.59 & 0.59 & 0.59 & 0.59 & 0.59 & 0.59 & 0.58 & 0.58 \\
\hline .16 & 0.63 & 0.62 & 0.62 & 0.62 & 0.61 & 0.61 & 0.61 & 0.61 & 0.61 & 0.61 \\
\hline .17 & 0.65 & 0.65 & 0.64 & 0.64 & 0.64 & 0.64 & 0.64 & 0.64 & 0.63 & 0.63 \\
\hline .18 & 0.68 & 0.67 & 0.67 & 0.67 & 0.66 & 0.66 & 0.66 & 0.66 & 0.66 & 0.66 \\
\hline .19 & 0.70 & 0.69 & 0.69 & 0.69 & 0.69 & 0.68 & 0.68 & 0.68 & 0.68 & 0.68 \\
\hline .20 & 0.72 & 0.72 & 0.71 & 0.71 & 0.71 & 0.71 & 0.70 & 0.70 & 0.70 & 0.70 \\
\hline .21 & 0.74 & 0.74 & 0.73 & 0.73 & 0.73 & 0.73 & 0.72 & 0.72 & ๑. 72 & 0.72 \\
\hline .22 & 0.76 & 0.76 & 0.75 & 0.75 & 0.75 & 0.75 & 0.74 & 0.74 & 0.74 & 0.74 \\
\hline .23 & 0.78 & 0.78 & 0.77 & 0.77 & 0.77 & 0.76 & 0.76 & 0.76 & 0.76 & 0.76 \\
\hline .24 & 0.80 & 0.80 & 0.79 & 0.79 & 0.79 & 0.78 & 0.78 & 0.78 & 0.78 & 0.78 \\
\hline .25 & 0.82 & 0.81 & 0.81 & 0.81 & 0.80 & 0.80 & 0.80 & 0.80 & 0.80 & 0.80 \\
\hline .26 & 0.83 & 0.83 & 0.83 & 0.82 & 0.82 & 0.82 & 0.82 & 0.81 & 0.81 & 0.81 \\
\hline .27 & 0.85 & 0.85 & 0.84 & 0.84 & 0.84 & 0.83 & 0.83 & 0.83 & 0.83 & 0.83 \\
\hline .28 & 0.87 & 0.86 & 0.86 & 0.85 & 0.85 & 0.85 & 0.85 & 0.84 & 0.84 & 0.84 \\
\hline .29 & 0.88 & 0.88 & 0.87 & 0.87 & 0.86 & 0.86 & 0.86 & 0.86 & 0.86 & 0.86 \\
\hline .30 & 0.89 & 0.89 & 0.89 & 0.88 & 0.88 & 0.88 & 9.87 & 0.87 & 0.87 & 0.87 \\
\hline .31 & 0.91 & 0.90 & 0.90 & 0.89 & 0.89 & 0.89 & 0.89 & 0.89 & 0.88 & 0.88 \\
\hline .32 & 0.92 & 0.91 & 0.91 & 0.91 & 0.90 & 0.90 & 0.90 & 0.90 & 0.90 & 0.90 \\
\hline .33 & 0.93 & 0.93 & 0.92 & 0.92 & 0.91 & 0.91 & 0.91 & 0.91 & 0.91 & 0.91 \\
\hline .34 & 0.94 & 0.94 & 0.93 & 0.93 & 0.93 & 0.92 & 0.92 & 0.92 & 0.92 & 0.92 \\
\hline .35 & 0.95 & 0.95 & 0.94 & 0.94 & 0.94 & 0.93 & 0.93 & 0.93 & 0.93 & 0.93 \\
\hline .36 & 0.96 & 0.96 & 0.95 & 0.95 & 0.95 & 0.94 & 0.94 & 0.94 & 0.94 & 0.94 \\
\hline .37 & $D .97$ & 0.97 & 0.96 & 0.96 & 0.95 & 0.95 & 0.95 & 0.95 & 0.95 & 0.95 \\
\hline .38 & 0.98 & 0.97 & 0.97 & 0.97 & 0.96 & 0.96 & 0.96 & 0.96 & 0.95 & 0.95 \\
\hline .39 & 0.99 & 0.98 & 0.98 & 0.97 & 0.97 & 0.97 & 0.96 & 0.96 & 0.96 & 0.96 \\
\hline .40 & 0.99 & 0.99 & 0.98 & 1.98 & 0.98 & 0.97 & 0.97 & 0.97 & 0.97 & 0.97 \\
\hline .41 & 1.90 & 0.99 & 0.99 & 0.99 & 0.98 & 0.98 & 0.98 & 0.98 & 0.97 & 0.97 \\
\hline .42 & 1.01 & 1.00 & 1.00 & 0.99 & 0.99 & 0.98 & 0.98 & 0.98 & 0.98 & 0.98 \\
\hline .43 & 1.01 & 1.00 & 1.00 & 1.00 & 0.99 & 0.99 & 0.99 & 0.99 & 0.98 & 0.98 \\
\hline .44 & 1.01 & 1.01 & 1.00 & 1.00 & 1.00 & 0.99 & 0.99 & 0.99 & 0.99 & 0.99 \\
\hline .45 & 1.02 & 1.01 & 1.01 & 1.00 & 1.60 & 1.00 & 0.99 & 0.99 & 0.99 & 0.99 \\
\hline .46 & 1.02 & 1.02 & 1.01 & 1.01 & 1.00 & 1.00 & 1.00 & 1.00 & 1.60 & 1.00 \\
\hline .47 & 1.02 & 1.02 & 1.01 & 1.01 & 1.01 & 1.00 & 1.00 & 1.00 & 1.00 & 1.00 \\
\hline .48 & 1.02 & 1.02 & 1.01 & 1.01 & 1.01 & 1.00 & 1.00 & 1.00 & 1.00 & 1.00 \\
\hline .49 & 1.03 & 1.02 & 1.02 & 1.01 & 1.01 & 1.00 & 1.00 & 1.00 & 1.00 & 1.00 \\
\hline .50 & 1.03 & 1.02 & 1.02 & 1.01 & 1.01 & 1.00 & 1.00 & 1.00 & 1.00 & 1.00 \\
\hline
\end{tabular}


Table 1 Continued

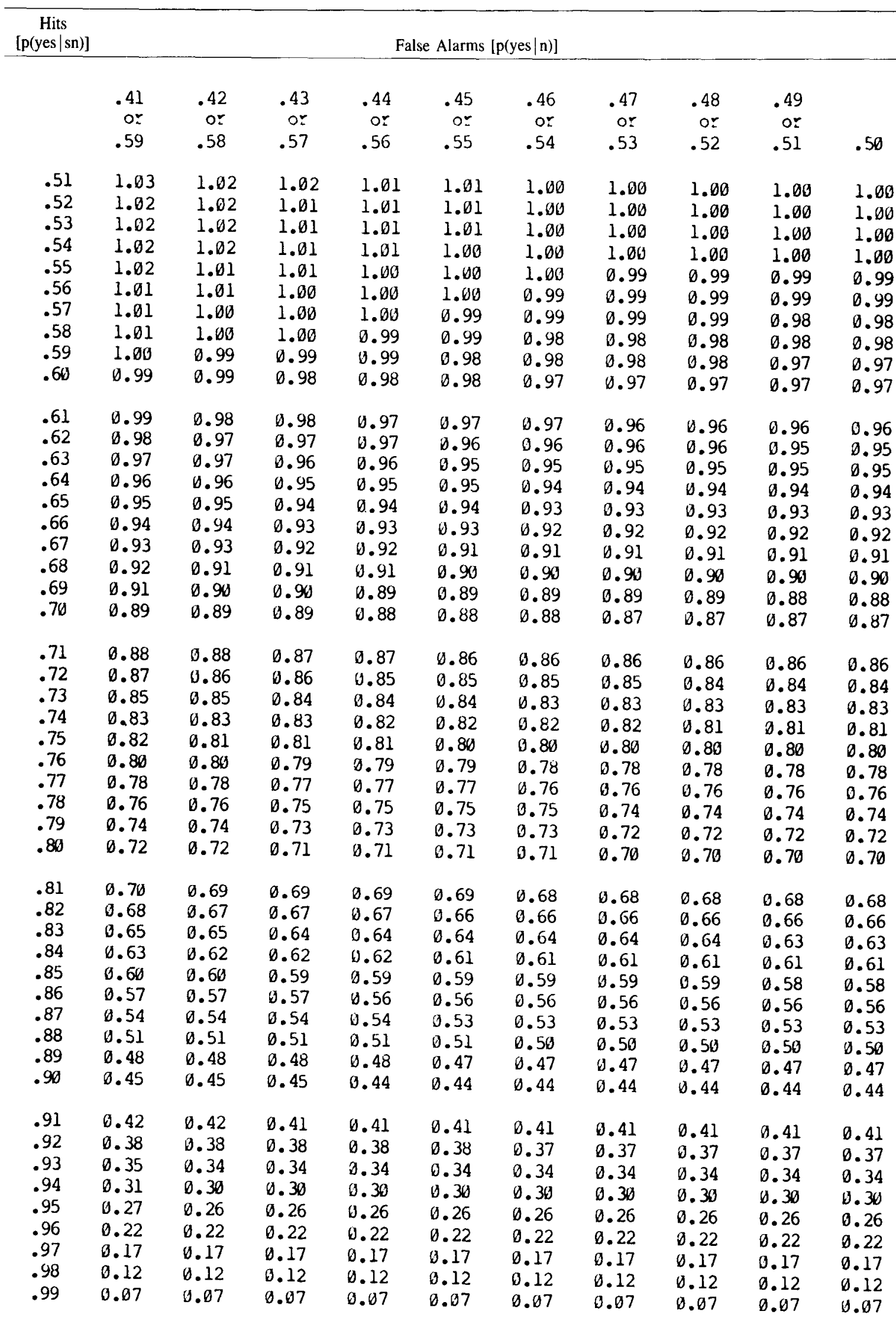


calls $B^{\prime}$. $B^{\prime}$ is calculated by the following formula, where $\mathrm{H}=$ hit rate and $\mathrm{FA}=$ false-alarm rate:

$B^{\prime}=[H(1-H)-F A(1-F A)] /[H(1-H)+F A(1-F A)]$.

The reader is cautioned to use these nonparametric measures in cases in which assumptions about the normal distribution of the $\mathrm{N}$ and $\mathrm{SN}$ curves and the assumption of homogeneity of variances are not satisfied.

The preceding table of $\beta$ values should serve as an aid to those investigators calculating several $\beta$ values from a yes/no task or determining $\beta$ values of successive points on ROC curves.

\section{REFERENCES}

Eluiot, P. B. (1964). Tables of d' . In J. A. Swets (Ed.), Signal detection and recognition by human observers. New York: Wiley.

Gescheider, G. A. (1976). Psychophysics: Method and theory. New York: Wiley.

Pollack, I. (1970). A nonparametric procedure for evaluation of true and false positives. Behavior Research Methods \& Instrumentation, $2,155-156$.

SNODGRASS, J. G. (1984). The pragmatics of measuring recognition memory performance. Unpublished manuscript, New York University, New York.

TANNER, W. P., \& SWets, J. A. (1954). A decision-making theory of visual detection. Psychological Review, 61, 401-409.

(Manuscript received September 21, 1983; revision accepted for publication July 30,1984 .) 